\title{
Ultrasonic Characterization of Newtonian and Non-newtonian Fluids
}

\author{
Md Sarowar Hossain ${ }^{1, *}$, Barnana Pal' ${ }^{2}$, P. K. Mukhopadhyay ${ }^{1}$ \\ ${ }^{1}$ LCMP, S.N. Bose National Centre for Basic Sciences, Salt Lake, India \\ ${ }^{2} \mathrm{CMP}$ Division, Saha Institute of Nuclear Physics, India
}

Copyright $\bigcirc 2018$ by authors, all rights reserved. Authors agree that this article remains permanently open access under the terms of the Creative Commons Attribution License 4.0 International License

\begin{abstract}
Viscous liquid causes a loss of acoustic energy for acoustic wave propagation through the liquid. From an acoustical point of view, Glycerin being Newtonian is a more complex medium as it is heterogeneous, anisotropic and viscoelastic. Non-Newtonian liquids like PEG-SiO ${\mathrm{DMF}-\mathrm{SiO}_{2}}_{2}$ solutions show shear-dependent nonlinear Viscosity. Apparent molar adiabatic compressibility $\left(\emptyset_{k}\right)$, as well as bulk moduli $(\mathrm{K})$ and apparent molar volumes $\left(\varnothing_{v}\right)$ of different glycerin-water solutions, are evaluated in the present investigation. At the lower concentration region for glycerin-water solutions apparent molar adiabatic compressibility $\left(\phi_{k}\right)$ varies linearly with the independent variable either molality or $\mathrm{m}^{1 / 2}$. The pulse-echo method has been followed to measure attenuation coefficient and sound velocity in these liquids at room temperature. This sound velocity has been compared with the measurement from high precision density and velocity meter (Anton Paar DSA $5000 \mathrm{M}$ for the same temperature. The measurement technique has been reported to quantify adiabatic compressibility as $10 \%$ glycerin in water was found to be $41.1 \times 10^{-11} \mathrm{~Pa}^{-1}(\mathrm{~K}=2.43 \mathrm{GPa})$ including $1.86 \mathrm{~dB} / \mathrm{cm} / \mathrm{MHz}$ of attenuation, less compared to pure water but the ultrasonic absorption coefficient $\left(\mu_{\mathrm{a}} \approx 0.53\right)$ of $10 \%$ glycerin in water is nearer to pure water whereas the Non-Newtonian fluids show higher attenuation $(>3 \mathrm{~dB} / \mathrm{cm})$ and higher absorption $\left(\mu_{\mathrm{a}} \approx 0.6\right)$.
\end{abstract}

Keywords Viscous Medium, Ultrasonic Velocity, Sound Attenuation, Adiabatic Compressibility, Non-newtonian Fluids

\section{Introduction}

Newtonian and non-Newtonian fluids are separated due to their viscosity behavior as a function of shear rate, stress, deformation history. Hence, viscosities, the adiabatic compressibility of any fluid is of great use in characterizing the structure and behavior of a fluid that is Newtonian or non-Newtonian in nature [1-3]. Additionally, the acoustic properties of a viscous medium (sound velocity and sound absorption) [4-8] contain unique microscopic information that cannot be obtained by other methods. Among the various acoustic properties measurement techniques, pulse-echo testing (PE) is an interesting engineering tool because of its wide range of capabilities, ease of use and an absence of damage to the system or material under study. Its non-destructive mode allows many functions [9-14] like Acoustic Emission Testing (AET), Electromagnetic Testing (ET), Acoustic Resonance Testing (ART), Infrared Testing (IT), Magnetic Particle Testing (MPT), Ultrasonic Testing (UT) and Radiographic Testing (RT) etc. The ultrasonic pulse-echo technique involves a mechanical pulse that is merely a stress a mechanical perturbation of the medium that propagates within it. The present work is an attempt to study both the Newtonian and Non-Newtonian liquids by the mechanical pulse of $1 \mathrm{MHz}$ at $250 \mathrm{C}$. The measured ultrasonic velocity $(u)$ has been implemented to derive the other parameters such as adiabatic compressibility $(\beta)$, relative compressibility $\left(\beta / \beta_{o}\right)$ and change in adiabatic compressibility $\left(\beta-\beta_{o}\right)$, apparent molal volume $\left(\phi_{v}\right)$, apparent molal compressibility $\left(\phi_{k}\right)$ and dynamic viscosity $(\eta)$. Hence, many important physical properties of the fluids may be easily to obtain.

\section{Materials and Methods}

High purity and analytical grade samples of Glycerin (AR $\geq 99.5), \quad \mathrm{N}, \quad \mathrm{N}$-Diethylformamide (AR $\geq 99$ ), Polyethylene Glycol (AR $\geq 99$ ), Ethylene Glycol (AR $\geq 99$ ), Ethanol and Cellulose microcrystalline powder procured from Merck specialties PVT. Ltd, Methylcellulose from Loba Chemie, Amylopectin from Tokyo chemical were used as raw materials. The mixtures were prepared on the percentage basis $(\mathrm{W} / \mathrm{W})$ from these chemicals. A piezoelectric transducer of $1 \mathrm{MHz}$ introduced mechanical pulses into the material under study. A reflector was placed parallel to the transducer face at a suitable distance to produce a series of echoes to analyze the specimens and the various interactions inside it. The experimental setup is shown in the Fig. 1. A DL1640 digital oscilloscope of the 
frequency range of $200 \mathrm{MHz}$ was used to study the amplitudes and the delay of each echo. The delay between two successive echoes was in microseconds that were used to measure the ultrasonic velocities. The unit conversion $\mathrm{cm}^{-1}$ to $\mathrm{dBcm}^{-1}$ was done as [10]

$$
\alpha\left[\mathrm{dBcm}^{-1}\right]=8.686 \alpha \mathrm{cm}^{-1}
$$

In this paper, the measurement of $\alpha$ was done by exponential fitting the echo amplitudes using the formula

$$
V=V_{o} e^{-\alpha Z}
$$

Where $\mathrm{V}$ is the echo amplitude in voltage and $\mathrm{Z}$ is the distance between two echoes in $\mathrm{cm}$. We measured the absorption coefficient, $\mu_{a}$ from the following equation.

$$
\mu_{a}=1-(B / A)^{2}
$$

Where A and B are the amplitudes of the incident and reflected waves. In fluids, the adiabatic bulk modulus of elasticity $K$ is reciprocal of the adiabatic compressibility $\beta$ in $\mathrm{Pa}^{-1}$ given by the relation,

$$
\beta=\frac{1}{u^{2} d}=\frac{1}{K}
$$

Where $d$ is the density of the medium in $\mathrm{kgm}^{-3}$ and $u$ is the velocity in $\mathrm{ms}^{-1}$ of the sound wave through that medium $K$ physically corresponds to the force opposing compression of the fluid. Compressibility is the relative change in volume when the pressure changes by one unit. We measured the speed of sound in liquids by the pulse-echo technique at nearly $25^{\circ} \mathrm{C}$. The formula, $v=2 l / t$ is used $(l$ is the distance that the wave traveled in time $t$ ). To standardize the velocity data for all samples a high precision density and velocity meter (Anton Paar DSA $5000 \mathrm{M}$ ) has been used for measuring both the density and speed of sound at $25^{\circ} \mathrm{C}$ where the uncertainties in density are within $\pm 5 \times 10^{-3} \mathrm{kgm}^{-3}$ under most favorable conditions.

Apparent molar volumes $\phi_{v}$ of the solutions was calculated using the following relation

$$
\emptyset_{v}=\frac{M}{d}+\frac{1000\left(d_{0}-d\right)}{m d d_{0}}
$$

Where $M$ is the relative molar mass of the solute, $m$ is the molality of the solution, and $d$ and $d_{0}$ are the densities of solution and solvent, respectively. The apparent molal volume varies with concentration following Masson's empirical relation [11]

$$
\phi_{v}=\phi_{v}{ }^{o}+S_{v} m^{1 / 2}
$$

Where $S_{v}$ gives constant value and $\phi_{v}{ }^{o}$ is denoted by the limiting apparent molal volume at infinite dilution. The apparent molar adiabatic compressibility $\emptyset_{k}$ of liquid solutions was calculated from the relation

$$
\emptyset_{k}=\frac{1000}{m d_{o}}\left(\beta-\beta_{o}\right)+\emptyset_{v} \beta
$$

Where $\beta$ and $\beta_{o}$ are the compressibility coefficients of the solvent and solution, respectively. $\phi_{k}$ Is also a function of $m$ obtained by Gucker [10] from Debye Hückel theory [12] and is given by

$$
\phi_{k}=\phi_{k}{ }^{o}+S_{k} m^{1 / 2}
$$

\begin{tabular}{|c|c|c|c|}
\hline Non- Newtonian Fluid & Heat & Stirring & Sonication \\
\hline $\begin{array}{c}50 \mathrm{ml} \mathrm{H}_{2} \mathrm{O}+\underset{ }{2.5 \mathrm{~g} \text { Carboxy-methyle }} \\
\text { Cellulose }\end{array}$ & $60^{\circ} \mathrm{C}$ & $\begin{array}{l}\text { 600rpm } \\
\text { for } 3 \mathrm{~h}\end{array}$ & $6 \mathrm{~h}$ \\
\hline $\begin{array}{c}\text { 75ml Polyethylene Glycol }+15 \mathrm{~g} \\
\mathrm{SiO}_{2}[\mathrm{P}]+20 \mathrm{ml} \text { Ethanol }\end{array}$ & $60^{\circ} \mathrm{C}$ & $\begin{array}{l}400 \mathrm{rpm} \\
\text { for } 1 \mathrm{~h}\end{array}$ & $9 \mathrm{~h}$ \\
\hline $\begin{array}{l}\text { 75ml Polyethylene Glycol }+10 \mathrm{~g} \\
\mathrm{SiO}_{2}[\mathrm{~F}]+20 \mathrm{ml} \text { Ethanol }\end{array}$ & $60^{\circ} \mathrm{C}$ & $\begin{array}{l}\text { 600rpm } \\
\text { for } 3 \mathrm{~h}\end{array}$ & $7 \mathrm{~h}$ \\
\hline $\begin{array}{c}\text { 20ml N,N-dimethyle Formamide } \\
+4 \mathrm{~g} \text { Carboxy-methyle } \\
\text { Cellulose }+1.3 \mathrm{~g} \mathrm{SiO}_{2}[\mathrm{~F}]\end{array}$ & $60^{\circ} \mathrm{C}$ & $\begin{array}{l}\text { Not } \\
\text { done }\end{array}$ & $5 \mathrm{~h} 30 \mathrm{~min}$ \\
\hline $\begin{array}{l}20 \mathrm{ml} \mathrm{N}, \mathrm{N} \text {-dimethyle Formamide } \\
+1 \mathrm{~g} \text { Methyl Cellulose }+0.8 \mathrm{~g} \mathrm{SiO}_{2}[\mathrm{~F}]\end{array}$ & $60^{\circ} \mathrm{C}$ & $\begin{array}{l}\text { 600rpm } \\
\text { for } 3 \mathrm{~h}\end{array}$ & $9 \mathrm{~h}$ \\
\hline $\begin{array}{c}\text { 30ml } \mathrm{H}_{2} \mathrm{O}+2 \mathrm{~g} \text { Carboxy-methyle } \\
\text { Cellulose }+8 \mathrm{~g} \mathrm{SiO}_{2}[\mathrm{P}]\end{array}$ & $60^{\circ} \mathrm{C}$ & $\begin{array}{l}\text { 600rpm } \\
\text { for } 3 \mathrm{~h}\end{array}$ & $10 \mathrm{~h}$ \\
\hline $\begin{array}{c}\text { 50ml Polyethylene Glycol }+3 \mathrm{~g} \\
\text { Carboxy-methyle Cellulose }+5 \mathrm{~g} \\
\mathrm{SiO}_{2}[\mathrm{P}]\end{array}$ & 30 & $\begin{array}{l}\text { 600rpm } \\
\text { for } 2 \mathrm{~h}\end{array}$ & $5 \mathrm{~h} 30 \mathrm{~min}$ \\
\hline $\begin{array}{c}30 \mathrm{ml} \mathrm{H}_{2} \mathrm{O}+1.29 \mathrm{~g} \text { Carboxy-methyle } \\
\text { Cellulose }+5 \mathrm{~g} \text { Amylopectin }\end{array}$ & 30 & $\begin{array}{l}\text { Not } \\
\text { done }\end{array}$ & $7 \mathrm{~h}$ \\
\hline $\begin{array}{c}\text { 40ml Ethylene Glycol }+1 \mathrm{~g} \\
\text { Carboxy-methyle Cellulose }+3 \mathrm{~g} \\
\mathrm{SiO}_{2}[\mathrm{~F}]+5 \mathrm{ml} \text { Ethanol }\end{array}$ & $60^{\circ}-80^{\circ} \mathrm{C}$ & $\begin{array}{l}\text { 600rpm } \\
\text { for } 6 \mathrm{~h}\end{array}$ & $7 \mathrm{~h}$ \\
\hline
\end{tabular}

where $S_{k}$ is a constant value and $\phi_{k}{ }^{0}$ is the limiting apparent molal volume at infinite dilution.

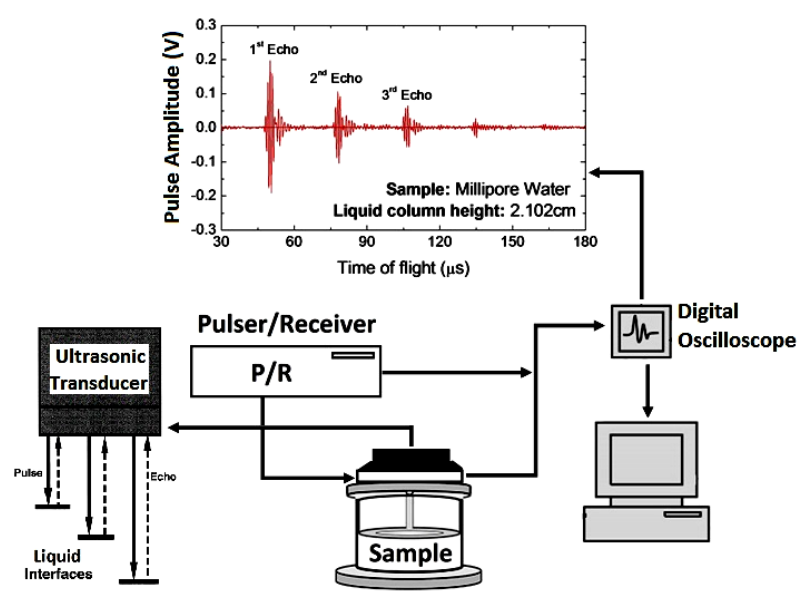

Figure 1. Schematic diagram of experimental setup

\section{Experimental Procedure}

\subsection{Preparation of Samples}

Table1. Sample preparations

Both the Newtonian and Non-Newtonian fluids have been studied by the attenuation coefficient, sound velocity, adiabatic compressibility as well as bulk modulus. Different concentrations of $99.5 \%$ pure glycerin in distilled water were used as the Newtonian fluids. The other types of fluids i.e. Non-Newtonian have been prepared by the 
stirring and sonication process including heat treatment at $60 \mathrm{oC}$ to $80 \mathrm{oC}$ as shown in Table 1

\section{Result and Discussion}

A typical case of pulse-echo with the envelopes, each was captured from the oscilloscope screen and shown in Fig. 2A and Fig. 2B. Best exponential decay fitting ( $R 2=0.999)$ was found for the liquid column height of $0.74 \mathrm{~cm}$. Hence overall behavior of echoes as a function of different concentrations of glycerin in water (Fig. 2B) was chosen for a fixed liquid column height of $0.74 \mathrm{~cm}$
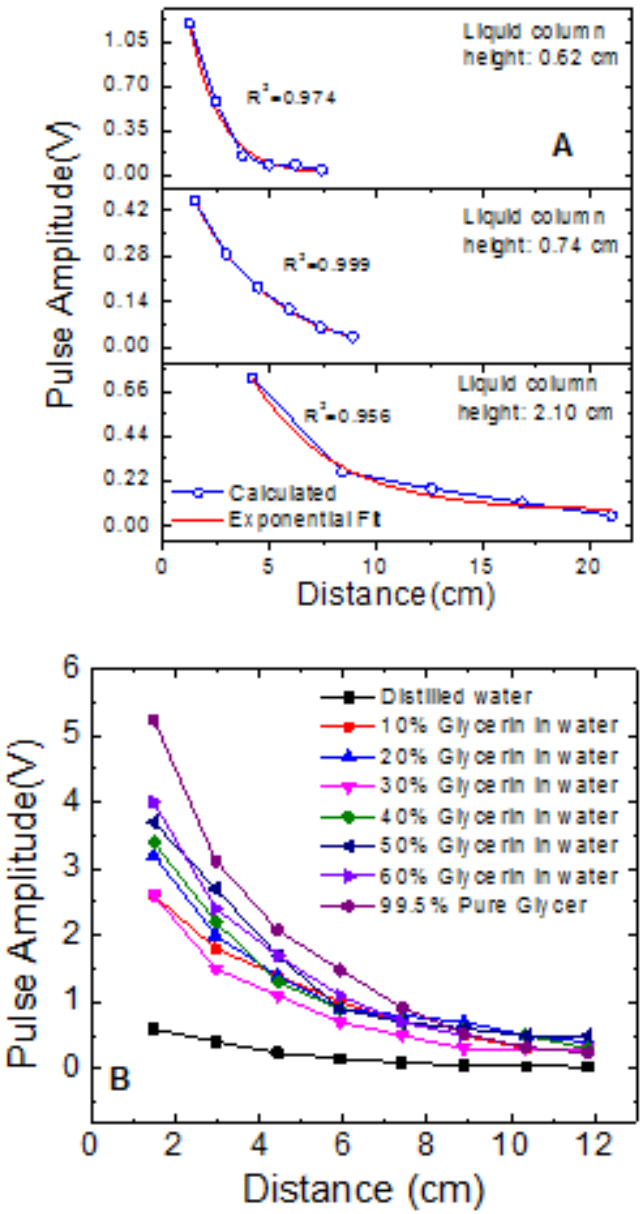

Figure 2. (A) Exponential decay of pulse amplitudes in different liquid column height and (B) Ultrasonic attenuation in various percentage of glycerin in water by pulse echo techniques

Ultrasonic velocity of $1 \mathrm{MHz}$ for different concentrations of glycerin solution was determined from the pulse-echo method using a single transducer. These data were compared with those from the high precision Density and Sound Velocity Meter, model DSA $5000 \mathrm{M}$ at the same temperature. In Fig. 3 we compared these data from the two techniques and they were found to be very close. Increases in sound velocity as a function of different concentrations come from changes in compressibility in the medium. This also affected the attenuation of sound waves in those different media. Ultrasound attenuation is characterized by the exponential decrease of the maximum peak values, i.e. amplitude (v) with the travelling distance $\mathrm{z}$.

Table 2. Parameters of various concentration of glycerin measured by $\mathrm{PE}$ at $25 \mathrm{oC}$ where $\rho$ is density, $\eta$ is viscosity, $v_{1}$ and $v_{2}$ is the sound velocities measured by pulse echo technique and high precision apparatus Anton Paar DSA 5000M respectively

\begin{tabular}{cccccc}
\hline $\begin{array}{c}\text { Glycerin } \\
\text { solution in } \\
\text { water }\end{array}$ & $\begin{array}{c}\boldsymbol{\rho} \\
\left(\mathbf{g m} / \mathbf{c m}^{\mathbf{3}}\right)\end{array}$ & $\begin{array}{c}\boldsymbol{\eta} \\
(\mathbf{M P a . s})\end{array}$ & $\begin{array}{c}\boldsymbol{\alpha} \\
(\mathbf{d B} / \mathbf{c m})\end{array}$ & $\begin{array}{c}\boldsymbol{v}_{\mathbf{1}} \\
(\mathbf{m} / \mathbf{s})\end{array}$ & $\begin{array}{c}\boldsymbol{v}_{2} \\
(\mathbf{m} / \mathbf{s})\end{array}$ \\
\hline $\mathbf{0 \%}$ & 0.99 & 0.8752 & 2.57 & 1488.51 & 1482.32 \\
$\mathbf{1 0 \%}$ & 1.03 & 1.3754 & 1.86 & 1523.53 & 1537.71 \\
$\mathbf{2 0 \%}$ & 1.05 & 1.7639 & 3.1 & 1569.70 & 1579.88 \\
$\mathbf{3 0 \%}$ & 1.07 & 2.4232 & 3.17 & 1593.85 & 1624.63 \\
$\mathbf{4 0 \%}$ & 1.08 & 2.65 & 3.15 & 1670.97 & 1652.34 \\
$\mathbf{5 0 \%}$ & 1.10 & 3.56 & 2.75 & 1698.36 & 1678.78 \\
$\mathbf{6 0 \%}$ & 1.11 & 4.42 & 2.77 & 1726.67 & 1705.67 \\
$\mathbf{1 0 0} \%$ & 1.26 & 945.95 & 4.75 & 1927.08 & 1920 \\
\hline
\end{tabular}

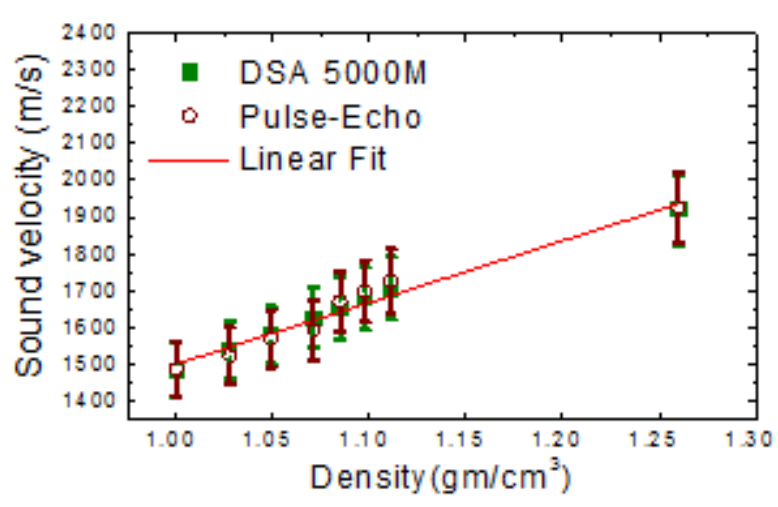

Figure 3. Sound velocities measured by DSA 5000M and PE technique as a function of densities for glycerine water mixture.

We calculated the attenuation coefficient, $\alpha$ from the exponential fitting shown in Fig. 3A. The fitting followed the following equation:

$$
V=V_{o}+A e^{-\frac{z}{t}}=V_{o}+A e^{-\alpha z}
$$

Where $V_{o}$ and $V$ are the amplitudes of pulse 1 and 2 respectively. Distances were calculated from $(\mathrm{z}=2 \times$ echo number $\times$ liquid column height) .Time gap between two successive pulses was used to calculate the sound velocities. Fig. 4A shows the analysis of attenuation coefficient and viscosity as a function of weight percent of glycerin in $\mathrm{H}_{2} \mathrm{O}$. Attenuation varies with the concentration as well as viscosity also. Sound absorption is by three ways that is viscous losses, thermal elastic damping and mechanical damping in the material itself. Fig. 4B shows the sound attenuation anomaly for the different types of non-Newtonian fluids. Viscosities of all Non-Newtonian fluids are not constant and show the dependency on the shear rate. 

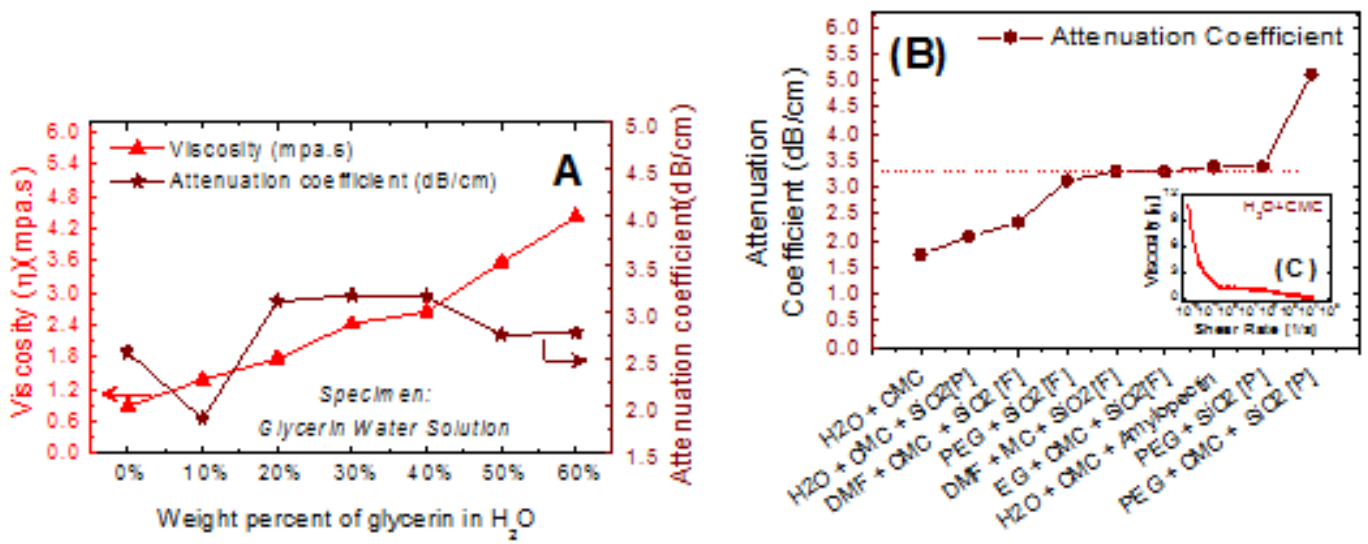

Figure 4. Ultrasonic attenuation and viscosity (A) in different weight percentage of $\mathrm{C}_{3} \mathrm{H}_{8} \mathrm{O}_{3}$ (B) in different non-Newtonian fluids and (C) inset showing nonlinearity of viscosity in $\mathrm{H}_{2} \mathrm{O}+\mathrm{CMC}$
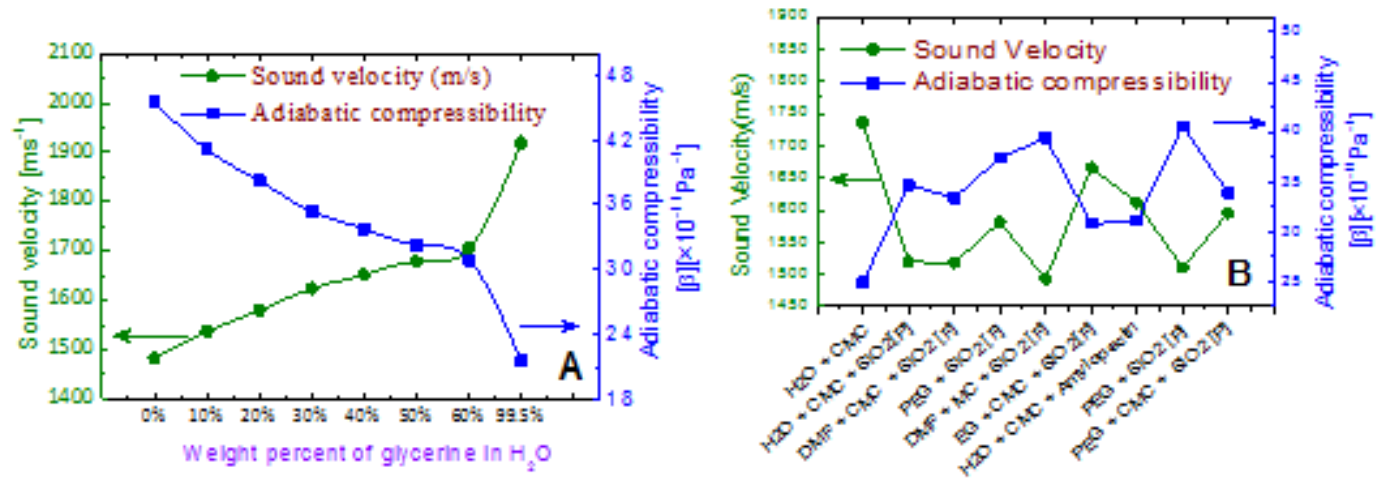

Figure 5. (A) Ultrasonic velocity and adiabatic compressibility for (B) different weight percent of $\mathrm{C}_{3} \mathrm{H}_{8} \mathrm{O}_{3}$ (b) different types of non-Newtonian fluids

Table 3. Molal concentration, Apparent molar volumes, Adiabatic compressibility coefficients, apparent molar adiabatic Compressibility, $\Delta \beta$ and $\Delta \beta / \beta_{o}$ for glycerin solution.

\begin{tabular}{|c|c|c|c|c|c|}
\hline $\mathrm{m}(\mathrm{mol} / \mathrm{kg})$ & $\emptyset_{v}\left(\mathbf{M}^{3} \mathbf{m o l}^{-1}\right)$ & $\beta\left(\mathrm{kg}^{-1} \mathrm{~ms}^{2}\right.$ or $\left.\mathrm{Pa}^{-1}\right)$ & $\emptyset_{k}\left(\mathrm{M}^{3} \mathrm{~mol}^{-1} \mathrm{~Pa}^{-1}\right)$ & $\Delta \boldsymbol{\beta}\left(\mathbf{P a}^{-1}\right)$ & $\Delta \boldsymbol{\beta} / \boldsymbol{\beta}_{o}$ \\
\hline 1.21 & 0.07 & $41.15 \times 10^{-10}$ & $-8.46 \times 10^{-12}$ & $-4.36 \times 10^{-11}$ & -0.09 \\
\hline 2.71 & 0.07 & $38.19 \times 10^{-11}$ & $2.35 \times 10-15$ & $-7.32 \times 10^{-11}$ & -0.16 \\
\hline 4.65 & 0.07 & $35.37 \times 10^{-11}$ & $3.59 \times 10-12$ & $-1.01 \times 10^{-10}$ & -0.22 \\
\hline 7.24 & 0.07 & $33.77 \times 10^{-11}$ & $8.81 \times 10-12$ & $-1.17 \times 10^{-10}$ & -0.26 \\
\hline 10.86 & 0.08 & $32.33 \times 10^{-11}$ & $1.23 \times 10-11$ & $-1.32 \times 10^{-10}$ & -0.29 \\
\hline 16.29 & 0.08 & $30.94 \times 10^{-11}$ & $1.48 \times 10-11$ & $-1.46 \times 10^{-10}$ & -0.32 \\
\hline 108.04 & 0.07 & $21.53 \times 10^{-11}$ & $1.31 \times 10-11$ & $-2.40 \times 10^{-10}$ & -0.52 \\
\hline
\end{tabular}

For the glycerin-water mixtures, the decrease in compressibility attributes by the formation of flexible $\mathrm{H}$-bond in the aqueous medium. This results in decrease in sound velocity in the higher dilution limit. For pure glycerin the adiabatic compressibility shows $21.53 \times 10^{-11}$ and by using the speed of sound value measured by the pulse-echo technique which is comparable to a standard value. It may be of interest to note that $\mathrm{v}$ is linear in concentration only in the dilute limit, mimicking a Vegard's law behavior for binary mixtures. However, it breaks down in the concentrated region $(>60 \%)$, showing nonlinearity in the behavior. The negative values of $\Delta \beta$ and $\Delta \beta / \beta_{O}$ is due to the solute - solvent interaction [13-14]. Increase in $\Delta \beta$ and $\Delta \beta / \beta_{O}$ values with an increase in glycerin concentration may be attributed to an overall increase in the cohesive forces in the solutions [15]. In addition, the successive increase of glycerin concentration in the water enhances the incompressible part of the solution.

The Apparent molar volumes $\left(\varnothing_{v}\right)$ for all compounds in aqueous solution were calculated using eq. [1.6] and the data shown in the table 3 . 

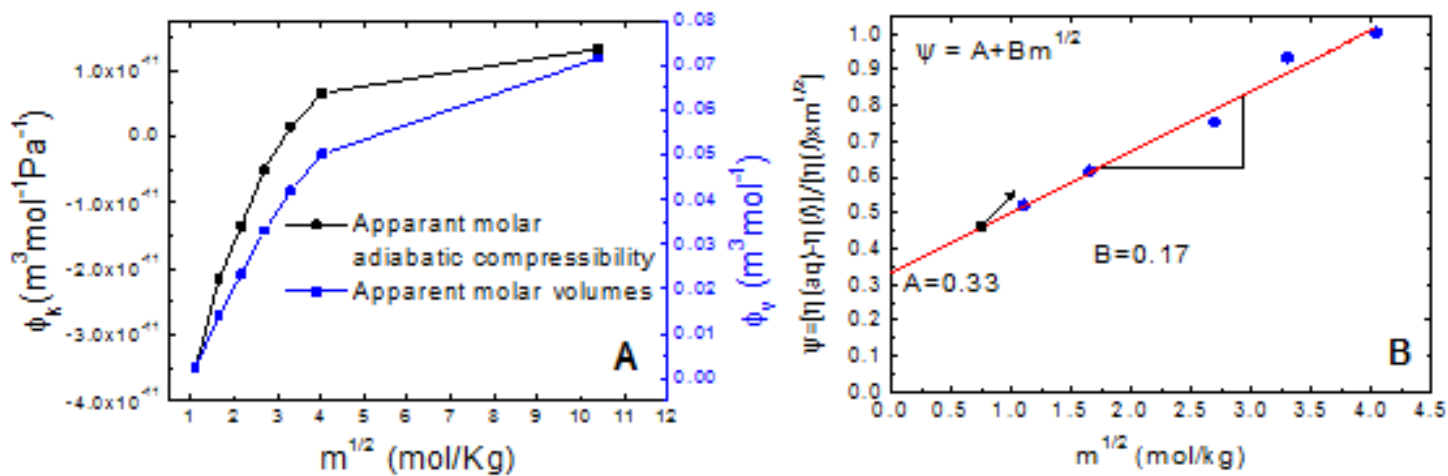

Figure 6. (A) Apparent molar volumes and apparent molar adiabatic compressibility as a function of concentration (m) for glycerin solution and (B) $\Psi$ vs square root of the molal concentration, $\mathrm{m}$ in glycerin water solutions

The variation of apparent molar volumes $\left(\phi_{v}\right)$ and apparent molar adiabatic compressibility $\left(\phi_{k}\right)$ in the glycerin- water mixtures as a function of concentration is shown in Fig. 6. A tendency to assume linear relation between the apparent molar adiabatic compressibility $\left(\phi_{k}\right)$ and the independent variable either molality or $\mathrm{m} 1 / 2$, is seen to hold in the lower concentration region. $\Psi$ as a function of the square root of molal concentration of glycerin has intercept A and slope B is calculated using Jones-Dole equation. "A" coefficient describes the impact of charge-charge interactions on the viscosity of a solution and estimated using the Falkenhagen equation [16]. The value of " $\mathrm{A}$ " is generally positive for the electrolyte solutions and we got the positive value 0.33 for the different concentrations of glycerine in water. The positive value 0.17 for " $\mathrm{B}$ " coefficient indicates a tendency for the solute to enhance water-water interactions and thus raise the viscosity and the solute is a structure former. [17]

\section{Conclusions}

The attenuation ultrasonic wave through a fluid results from several mechanisms of absorption. At the beginning of the propagation, the absorption is mainly due to the viscosity. Each layer of the fluid tends to slow down the displacement of the adjacent layers, causing thus the damping of the wave as it penetrates into the fluid. In these samples, which are Non-Newtonian viscous fluid contains a polymer (like cellulose or starch) along with very small molecular particles and Silica of diameter $\sim 0.3$ micron. So while passing through these solutions the ultrasonic waves loses its energy in two ways- one, to propagate through this solution the sound wave has to work to produce mechanical vibrations on those large chains of Cellulose or Starch polymers, Second, while travelling through this chemical the sound wave is scattered by the silica micro-particles. The propagation of sound waves through this special type of solutions involves displacement of positions of molecules, which in turn increases the potential energy of those molecules in expense of sound energy and this internal energy has a little contribution to the thermal agitations to the samples. With our present setup of pulse-echo method, we deduce a lot of information about internal parameters of the fluid. We have done the measurements at a fixed temperature only, however, the experiment as a function of temperature will give more details of sample properties like activation energy for structural relaxation etc.

\section{REFERENCES}

[1] Frank J., Millero, Antonio L., Surdo and Charles Shin, The apparent molal volumes and adiabatic compressibilities of aqueous amino acids at $25^{\circ} \mathrm{C}$, J. Phys. Chem., 82, (1978) 784-792.

[2] Sumathi T. and Varalakshmi M., Ultrasonic velocity, density and viscosity measurement of methionine in aqueous electrolytic solutions at 303k, J. Chem., 3 (2010) 550-555

[3] Alexander Apelblat, Thermodynamic Properties of Aqueous Electrolyte Solutions. Compressibility Studies in $0.1,0.5$ and $1.0 \mathrm{~mol} \cdot \mathrm{kg}-1$ Lithium Chloride Solutions at Temperatures from 278.15 to 323.15 K, J. Sol. Chem., 36 (2007) 1437-1456

[4] Hartmann B, Jarzynski J. Immersion apparatus for ultrasonic measurements in polymers. J. Acoust. Soc. Am., 1974. Vol.56. No.5. P.1469-1477.

[5] Ping He. Measurement of acoustic dispersion using both transmitted and reflected pulses. J. Acoust. Soc. Am., 2000. Vol.107 No.2. P.801-807.

[6] Wróbel G., Wierzbicki Ł. Ultrasonic methods in diagnostics of polyethylene. Archives of Materials Science and Engineering, 2007, Vol.28. Issue 7, P.413-416.

[7] Raišutis R., Kažys R., Mažeika L. Application of ultrasonic characterization methods for highly attenuating plastic materials. NDT \& E International. 2007, Vol. 40, P. 324-332.

[8] Raichel DR (2006). The science and applications of acoustics. Springer Science \& Business Media

[9] Batra N. K. and Chaskelis, H. H., Determination of 
Minimum Flaw Size Detectable by Ultrasonics in Titanium Alloy Plates, NDT International. 18 (1985) 261-264.

[10] Cerri M. and Cusolito R. Experiences in Capability Assessment of Manual Ultrasonic Examination Techniques for Planar Flaw Detection and Sizing in Austenitic Stainless Steel Joints, NDT International. 22 (1989) 229-239

[11] Hislop J. D., Flaw Size Evaluation in Immersed Ultrasonic Testing, Non-Destructive Testing. 2 (1969) 183-192

[12] Marianeschi E. and Tili T., A Note on the Smallest Defect that can be Detected using Ultrasonics, NDT International, $16,(1983) 75-77$

[13] Turó A., Chávez J. A., García-Hernández M. J., Bulkai A.,
Tomek P., Tóth G., Gironés A. and Salazar J. Ultrasonic Inspection System for Powder Metallurgy Parts." Measurement. 46 (2013) 1101-1108

[14] Lakes and Roderic S., Viscoelastic Solids. CRC Press, (1998).

[15] Pandey D. and Akhatar Y., Ultrasonic studies of aqueous concentrated electrolyte solutions at 298.15 K, J. Pure Appl. Ultrason., 18 (1996) 108

[16] Harned H. S. and Owen B. B., The Physical Chemistry of Electrolyte Solutions, Reinhold, New York, 1958.

[17] Nightingale E.R., Phenomenological Theory of Ion Solvation Effective Radii of Hydrated Ions, J. Phys. Chem. 63 (1959) 1381-138 